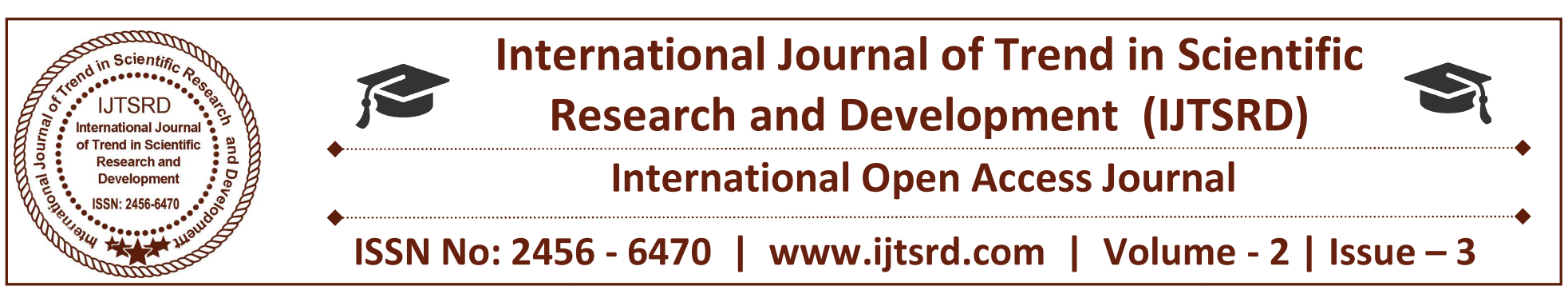

\title{
Heat Transfer Enhancement by Using Dimple Surface
}

\author{
Nadeem Pathan*, Sanjay Mitkari \\ Department of Mechanical Engineering, G.H. Raisoni College of Engineering and Management, \\ Pune, Maharashtra, India
}

\begin{abstract}
Nowadays, to improve or to increase efficiency of heat transfer medium to another task is very difficult to find new method by using geometrical changes and some using the some external sources affected on heat transformation dimple surfaces in which the dimple is nothing but "hole" like or depression like structure in which air circulation is created and to help heat transfer more effectively, we create some shapes of dimple; and to this various shapes we compare with flat plate then we find some changes in our data. We can use various methods to identify which shape is more effective.
\end{abstract}

Keyword: Dimple surfaces, layout and geometry, various shapes and arrangements

\section{INTRODUCTION}

The challenging task is to produce more effective and efficient heat transfer devices i.e. heat exchanger, to find the methods, which gives more information about the traditional method and to increase the heat transfer rate. To study heat transfer method industry and to increase the heat related factor such as heat transfer equipment and heat exchangers. To challenge some find new shapes and low cost and less material consumption which can be mainly considered. These visions are considered in our project.

Our project mainly depends on dimple surface, in which some geometrical changes have been done to increase heat transfer rate. We can mainly consider heat enhancement method in which mainly three methods are studied; and they are: active method, passive method and combine method.

1. In active method, some geometrical changes are made to increase the heat transfer rate.

2. In passive method, some external sources are used to that source which helps to increase heat transfer rate.

3. Combine method is a combination of active and passive method in which some geometrical changes and some external sources can be used and to increase the heat transfer rate.

In our project, we can use combine method in which plate is considered and modified the plate with some geometrical changes and compared with flat plate; and also external sources like blower which gives air flow to the dimple plate have been used. After some analytical study, we can find out the difference between the flat plate and the dimple plate calculation and then we conclude that which dimple surface is more effective to increase the heat transfer rate and then we identify that the method used in our project is right or wrong. Mainly, we used two different mediums to transfer the heat in our project, we can say cold air and heat is the second medium to heat transfer; then our project is like heat exchanger and we can calculate the air distribution and project identification to increase heat transfer rate. Now calculation is done on heat transfer basis and all geometries are considered like various shapes and depth to height ratios. After studying previous papers, we concluded that the height to depth ratio matters to increase heat transfer rate.

This upper Figure 1 is flow phenomenon which mainly focuses on increasing the air circulations in the dimple surface. Air is passing dimple some air is 
passing in that dimple and air circulation is created due to this boundary layer get disturb and vortex is form in Marathi vortex is called as "BHOVRA". The air circulation formed inside the dimples results in reducing and disturbing the thermal boundary layer and to helps the transfer the heat under the layer of dimple surfaces.

Each dimple acts as a "Vortex Generator" which provides an intensive and stable heat and mass transfer between the dimpled surface and gaseous heating/cooling media, taking advantages of vortex heat transfer enhancement (VHTE), suppose air is passed to dimple (Figure 2).

\section{Research Gap}

$>$ Most of the papers $[1,2,3,5,7$ and 8] are related to the dimple geometry and modification and its applications to their uses.

$>$ No paper is related to how to improve heat transfer rate by dimple and its various types.

$>$ Main objective of our paper is to improve heat transfer rate by using dimple surfaces.

$>$ In this paper, we focus to obtain maximum heat transfer rate.

$>$ We used various geometrical changes done by our side.

\section{METHODOLOGY USED}

$>$ Study the different methods used in our project.

$>$ To study various shapes and geometries in our project.

$>$ To use advanced technology for heat enhancement.

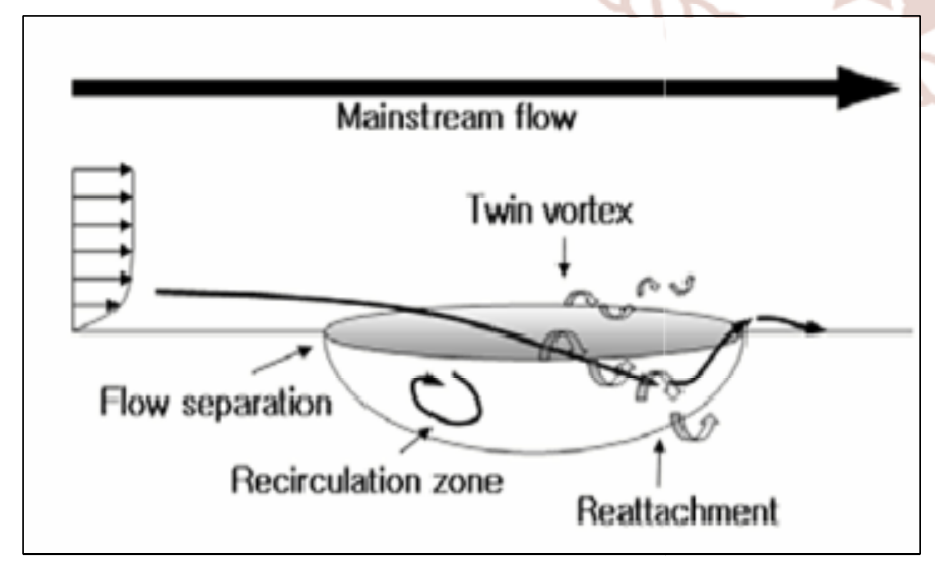

Fig. 1: Dimple Induced Flow Phenomenon

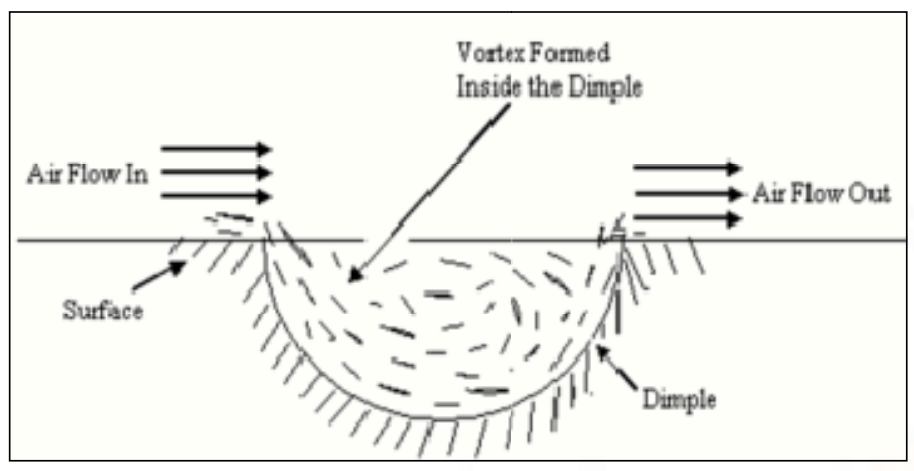

Fig. 2: Vortex Heat Transfer Enhancement Mechanism

\section{MATERIAL USED AND GEOMETRY SELECTION}

In our project, we selected the aluminium material and this material is reliable to various dimple shapes and then we selected total four shapes to the plate of aluminium whose dimensions are $405 \mathrm{~mm} \times 195 \mathrm{~mm}$ and the distance between the dimples is $15 \mathrm{~mm}$ each, to that parameter considered and then dimple plate is designed in its own way.

Test plate is of aluminum sheet of thickness $6 \mathrm{~mm}$ and having dimensions as $405 \mathrm{~mm} \times 195 \mathrm{~mm}$. The dimples produced on the test plate are of $8 \mathrm{~mm}$ diameter and $3 \mathrm{~mm}$ depth. For rectangular pattern arrangement, total 7 rows are employed in the stream wise direction and 14 columns are in span-wise direction. Test section coordinate system is employed for the study. Note that $\mathrm{Y}$ coordinate is normal to the test surface and $\mathrm{X}$ coordinate system is along flow $\mathrm{d}$ direction. The spacing in between center of two dimples in $\mathrm{X}$ and $\mathrm{Y}$ direction is of $11 \mathrm{~mm}$ up to highlight all of the above affiliation lines. Figure 3 shows the test with dimple section.

We can use total "9" numbers of plates in which we selected four shapes those are triangular dimple, trapezoidal dimple plate, oval shape dimple plate, and square shape dimple plate. And all plates are arrange inline and staggered shapes and all dimple plates are compared to flat plate which is made by aluminum. In case of in-line arrangement, all the dimples are in straight way and normally the all dimples are in straight line; and in the staggered arrangement, the dimple arrangement is mainly in the zigzag way. Some papers identify the in-line arrangement as less effective than the staggered arrangement. Because of that we compared flat plate, in-line arrangement plate and staggered arrangement 
plate and then concluded that which plate has the maximum heat transfer rate.

In general, we can use one important part which is known as flow strainer and this part is mainly used to transfer equal amount of air to each dimple and is called as flow straightener (Figure 4); and this equipment equally distributes the , passing air in all dimples; the passing air equally distribute by flow staightener of; in case the base of flow straightener is made of wooden plate to minimize the costing purpose, this device is used for long term and is very effective to transfer the flow in straight way and the equally distribute all the air passing is in equally manner in insulating box in which heater pate and the dimple plate available.
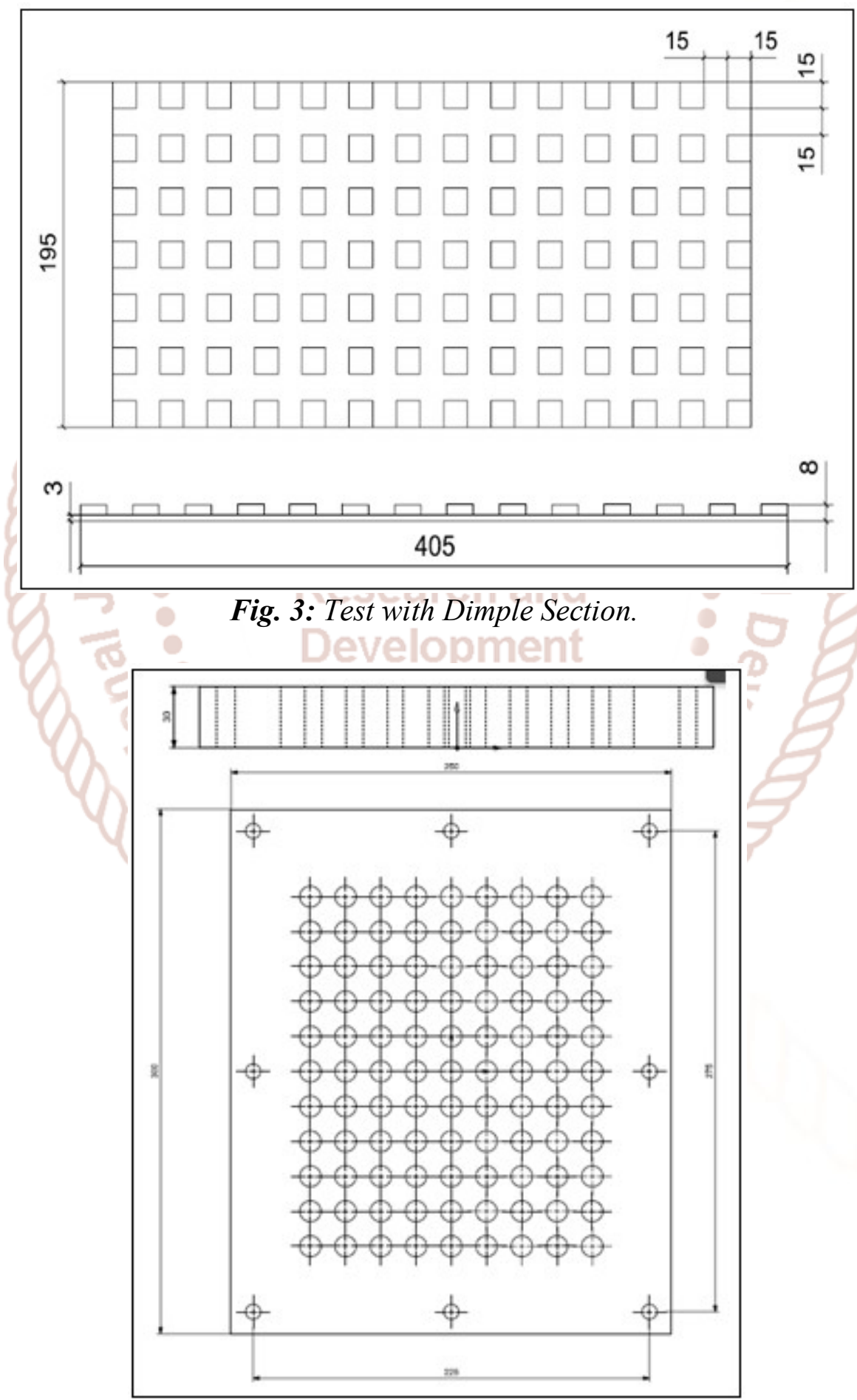

Fig. 4: Flow Straightener 
Dimension of the flow straightener is $300 \mathrm{~mm} \times 250 \mathrm{~mm}$ and is bigger than our test section plate fitted with convergent and divergent section.

\section{Layout of Project}

In case of the layout, we have used various equipment namely, blower, pressure control valve, pressure gauge, flow straightener, pipe, dimple plates, test section which is insulated box, thermo couple, various temperature sensors, digital temperature indicator and U-Tube manometer (Figure 5).

Steady state value of the plate and air temperatures in the channel, at various locations for a given heat flux and mass flow rate of air, is used to determine the values of performance parameters. The volume flow rate of air is determined from the pressure drop across the orifice meter, using a following relation: the useful heat gain of the air is calculated as: The heat transfer coefficient for the test section is calculated by Newton's law of cooling which states that, 'the rate of convection heat transfer is proportional to the temperature difference between surfaces of test plate and fluid.

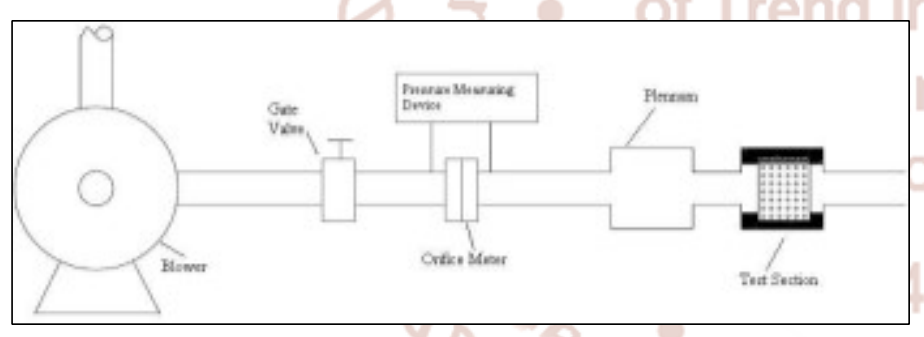

Fig. 5: Experimental Set Up.

\section{CONCLUSION}

1. The dimple depth to dimple print diameter $(\delta / \mathrm{D})$ ratios varying as $0.1,0.2$, and 0.3 , reported that at all Reynolds numbers considered, Nusselt number augmentations increase as dimple depth increases.

2. In a heat exchanger with the controlling thermal resistance on the tube side, it would always be possible to replace smooth tubes by selected dimpled tubes having only about one half of the length of the smooth tubes.

3. Spherical cavity dimple plate augment highest heat transfer rate than cylindrical cavity plate.

4. The heat transfer coefficient increases as the Reynolds number increases and the lower channel height cases show a higher heat transfer coefficient. Also, the heat transfer coefficient was higher for the densely distributed dimple case.

\section{REFERENCES}

1) Juin Chen, Hans Muller-Steinhagen, Duffy Geoffrey G. Heat Transfer Enhancement in Dimpled Tubes. Appl Therm Eng. 2001; 21(5): 535-547p.

2) Lutade PV, Khanwalkar PM, Kore SS, et al. Heat Transfer Enhancement by Jet Impingement on Dimpled Surface with Different Cavities. Mechanical Engineering Department, Sinhgad College of Engineering, University of Pune; 2005.

3) Wilfred Vinod Patrick. Computations of Flow Structures and Heat Transfer in a Dimpled Channel at Low to Moderate Reynolds Number. Thesis Submitted to the Faculty of the Virginia Polytechnic Institute and State University, Blacksburg, Virginia. 2005.

4) Fuguo Zhou. Studies on the Heat/Mass Transfer Characteristics and Fluid Structure in a Square Internal Cooling Channel with Dimpled Surfaces. A Thesis Submitted to the Graduate Faculty of the Louisiana State University; Aug 2007.

5) Somin Shin, Ki Seon Lee, Seoung Duck Park, et al. Measurement of the Heat Transfer Coefficient in the Dimpled Channel Effects of Dimple Arrangement and Channel Height. J Mech Sci Technol. 2009; 23(3): 624-630p.

6) Mohammad and Ligrani. Heat Transfer Augmentation Surfaces Using Modified Dimples/Protrusions. Dissertation Submitted to the Faculty of Virginia Polytechnic Institute and State University in Partial Fulfillment of the Requirement for the Degree of Doctor of Philosophy, Blacksburg, Virginia. 2008.

7) Kore Sandeep S, Joshi Satishchandra V, Kane Narayan S. Experimental Investigations of Heat Transfer Enhancement from Dimpled Surface in a Channel. International Journal of Engineering Science and Technology. Aug 2011; 3(8): 62276234p.

8) Patil Piyush V, Kumbhar DG. CFD Investigation on Dimpled Fins with Parameter Variation for Heat Transfer Augmentation. International Journal of Engineering Research \& Technology. Jun 2014 . 